Review Paper

\title{
Long-term quality of life among breast cancer survivors eligible for screening at diagnosis: a systematic review and meta-analysis
}

\author{
N. Moshina ${ }^{\text {a, }}{ }^{*}$, R.S. Falk ${ }^{\text {b }}$, S. Hofvind ${ }^{\text {a, c }}$ \\ ${ }^{a}$ Cancer Registry of Norway, Section of Cancer Screening, P.O. 5313, Majorstuen, Oslo, 0304, Norway \\ b Oslo University Hospital, Oslo Centre for Biostatistics and Epidemiology, P.O. 4950, Nydalen, Oslo, 0424, Norway

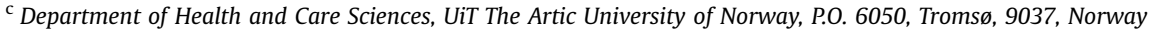

\section{A R T I C L E I N F O}

\section{Article history:}

Received 19 January 2021

Received in revised form

7 August 2021

Accepted 15 August 2021

Available online 21 September 2021

\section{Keywords:}

Breast neoplasm

Mass screening

Mammography

Survival

Quality of life

Review

\begin{abstract}
A B S T R A C T
Objectives: This study aimed to explore the long-term quality of life (QoL) among breast cancer survivors eligible for mammographic screening at diagnosis and compare that to QoL among women with no history of breast cancer.

Study design: Systematic review and meta-analysis.

Methods: A systematic review of randomised controlled trials and observational studies published between January 2000 and July 2019 was performed. Eight studies were included in the review. Six studies with QoL measurement scales $(0-100)$ were included in the meta-analysis. We used fixed and random effects models to obtain Cohen's d with $95 \%$ confidence interval (CI). Heterogeneity among studies was evaluated by the $\mathrm{I}^{2}$ statistics.

Results: Information about 6145 breast cancer survivors diagnosed between 1995 and 2012 and followed for >1-10 years was analysed. Four studies used SF-36/RAND-36, three studies used EORTC QLQ-C30, one study used FACT-G and one study used FACT-B. The mean score of QoL for breast cancer survivors varied from 63.0 (RAND SF-36, 0-100) to 110.5 (FACT-B, 0-123). Two studies showed better, three studies showed similar and two studies showed poorer mean scores for breast cancer survivors compared with women with no history of breast cancer. The meta-analysis showed no significant differences in QoL for breast cancer survivors compared with women with no history of breast cancer (Cohen's $\mathrm{d}=-0.07,95 \%$ confidence interval $[\mathrm{CI}]-0.14$ to 0.00 and $\mathrm{I}^{2}=83.7 \%$ for the fixed effect model; Cohen's $\mathrm{d}=-0.00,95 \% \mathrm{CI}-0.18$ to 0.17 and $\mathrm{I}^{2}=82.4 \%$ for the random effects model).

Conclusion: QoL did not differ between breast cancer survivors eligible for mammographic screening at diagnosis and followed for $>1-10$ years and women with no history of breast cancer.

( 2022 The Authors. Published by Elsevier Ltd on behalf of The Royal Society for Public Health. This is an open access article under the CC BY license (http://creativecommons.org/licenses/by/4.0/).
\end{abstract}

\section{Introduction}

Breast cancer is the most common cancer and cause of cancer death among women worldwide. ${ }^{1}$ Organised mammographic screening aims to reduce breast cancer mortality by detecting tumours at an early stage and decreasing the side-effects of treatment. ${ }^{2}$ Screening and improved treatment have been considered the main reasons for the increase in survival from breast cancer during the last decades. ${ }^{3,4}$ However, long-term side-effects of the treatment represent a major harm. ${ }^{5-13}$ Moreover, the detection of

\footnotetext{
* Corresponding author. Cancer Registry of Norway, P.O. 5313, Majorstuen, Oslo, 0304, Norway. Tel.: +4723333989.

E-mail addresses: Nataliia.Moshina@kreftregisteret.no (N. Moshina),rs@ous-hf.no (R.S. Falk), Solveig.Hofvind@kreftregisteret.no (S. Hofvind).
}

dormant and small, low proliferation tumours by screening brings another challenge to this secondary prevention because of the potential for overtreatment and accompanying long-term sideeffects. $^{14,15}$

Long-term quality of life (QoL) among breast cancer survivors has been evaluated in numerous studies, ${ }^{10,16-20}$ whereas the results from studies on women diagnosed with ductal carcinoma in situ or early-stage invasive breast cancer are limited. ${ }^{18,21,22}$ However, as far as we are aware, no studies based on individual data investigated long-term QoL among women with screen-detected breast cancer and women with no history of breast cancer. ${ }^{23,24}$ Therefore, the objectives of this review were to explore long-term QoL among breast cancer survivors eligible for mammographic screening at diagnosis between 1995 and 2018 and to compare the long-term QoL between these women and women with no history of breast 
cancer eligible for mammographic screening. Similar long-term QoL for women with screen-detected breast cancer and women with no history of breast cancer might imply that organised breast cancer screening and modern treatment positively affected the management and consequences of the disease.

\section{Materials and methods}

We carried out a systematic review of peer-reviewed papers published between January 2000 and July 2019. The Preferred Reporting Items for Systematic Reviews and Meta-Analyses guideline's checklist was used to ensure that relevant considerations were taken in all parts of the study. ${ }^{25}$

The long-term QoL was defined as perceived physical and mental health for $>1-10$ years since breast cancer diagnosis for breast cancer survivors or over a corresponding follow-up period for women with no history of breast cancer. A period of more than 1 year was chosen as a cutpoint for a long term, as we intended to include women with an early-stage breast cancer, which treatment, except the long-lasting hormonal therapy, might last less than 6 months and the effects of the treatment might be considered longterm effects for 14-18 months since diagnosis. ${ }^{26,27,28}$ QoL represented scores for general or global health scores ${ }^{29}$ obtained by various patient-reported outcome instruments (EORTC-QLQ-C30 [European Organization for Research and Treatment of Cancer Quality of Life Core Questionnaire], SF-36 [Short-Form Health Survey], FACT [Functional Assessment of Cancer Treatment Questionnaire], VAS [Visual Analogue Scale] and EQ-5D [EuroQual Questionnaire Five Dimensions]). Women residing in the countries where mammographic screening had been available since 1995 and the treatment of the disease had improved regardless of stage at diagnosis were considered eligible for screening. ${ }^{30,31}$ Women's age was not restricted, but women aged $45-75$ years were included in the analyses from the studies that performed stratification by age, as women of this age range are recommended mammographic screening. ${ }^{32}$ Furthermore, we restricted the search to early-stage breast cancer and the length of follow-up from $>1$ to 10 years since diagnosis or corresponding time frame for women with no history of breast cancer. Early-stage breast cancer included ductal carcinoma in situ, small invasive tumours $(<20 \mathrm{~mm})$ and/or earlystage invasive breast cancer (stages I and II).

\section{Literature search}

We conducted a search in MEDLINE, Embase, Google Scholar and Cochrane from 1 to 25 July 2019. We used the 'PICOS' (Population, Intervention, Comparison, Outcome and Study design) framework to identify the main terms for the literature search. ${ }^{33}$ The review aimed to explore the long-term $\mathrm{QoL}(\mathrm{O})$ among women with breast cancer $(\mathrm{P})$ who were eligible for mammographic screening (I). Women eligible for screening with no history of breast cancer was an optional criterion for comparison (C). Each search included a combination of the following terms: quality of life, treatment, treated, breast cancer, breast neoplasm, breast carcinoma, screening, screen-detected, mass screening and early detection. The combination of terms used is shown in Appendix A.

The study design included randomised controlled trials and observational studies. Systematic reviews and meta-analyses were used for literature check. Abstracts or poster presentations were not included. All titles of the identified papers were reviewed independently by N.M. and S.H. and discussed when the opinions were discordant (Fig. 1). The same authors read the abstracts of the papers with relevant titles and agreed on the papers that fulfilled the 10 criteria for inclusion in the review (Fig. 1). An additional optional criterion was inclusion of women diagnosed at the recommended screening age (45-75 years) if the differentiation of the results by age groups was performed. ${ }^{34}$ After reading the full text of the remaining papers, eight papers were included in the study. ${ }^{21,35-41}$

\section{Literature analysis}

For all included studies, data on aims, country and design, age and number of women studied and included in the review, data source, data collection method, month and year of breast cancer diagnosis, study period and coverage of organised breast cancer screening were extracted, tabulated and analysed (Table 1). Furthermore, data on breast cancer types/stages, long-term definition, comparison groups, methods to evaluate $\mathrm{QoL}$ and main findings were analysed (Table 2). Types and risk of biases in the studies were described in Appendix B, Table B1. The main results were defined as scores for QoL, including general or global health, and functioning scales. The scores were presented as means with standard deviation (SD), 95\% confidence interval (CI), or standard error (SE), based on the available data. The higher scores for QoL and all functioning scales corresponded to better QoL, whereas the higher scores for bodily pain corresponded to worse QoL. The mean scores for QoL were used to compare breast cancer survivors and the reference groups. The reference groups were defined as healthy women with no history of breast cancer, eligible for mammographic screening. ${ }^{21,35-40}$ The $P$ values for comparison in the included studies were two sided and were obtained using $t$-tests and unadjusted or age-adjusted analysis of variance. ${ }^{21,35-40}$ For the purpose of this review, all breast cancer survivors from the included studies were assumed to have screen-detected breast cancer, and women with no history of breast cancer were assumed either screening attendees or those who had attended screening and never been diagnosed with the disease.

We performed a meta-analysis for QoL assessed on scales 0-100 (EORTC-QLQ-C30 and SF-36) using fixed and random effects models. Two studies were excluded from meta-analysis; one study used FACT-G with a scale of $0-108$, and the other one did not have any comparison group. ${ }^{37-41}$ For each study included in the metaanalysis, Cohen's d effect size with 95\% CI and weights (percentage) was calculated as the mean difference between QoL scores for breast cancer survivors compared with the reference groups divided by the pooled SD; negative effect sizes reflected deficits compared with the reference groups. ${ }^{42}$ The results from the study by Klein et al. were used for the longest follow-up (10 years) performed with EORTC-QLQ-C30, as SF-36 was not considered breast cancer specific. ${ }^{39}$ Solely crude scores for QoL were included in the meta-analysis from Klein et al. Information on SD for the mean score from the studies by van Gestel et al. and Koch et al. was imputed using predictive mean matching for a continuous variable. $^{36,40}$ Statistical heterogeneity among studies was assessed through the $I^{2}$ statistics, where a value of $\geq 75 \%$ was interpreted as high heterogeneity. ${ }^{43}$ The funnel plot was used to estimate smallstudy effects. A $P$ value of $<0.05$ was considered statistically significant. All statistical analyses were performed using STATA/MP 16.0 (College Station, TX). The quality of the included studies was assessed according to the Cochrane guidelines and the CONSORTPRO criteria, and the main limitations were presented. ${ }^{44,45}$

\section{Results}

A total of 1558 papers were identified, whereas 1459 were excluded due to irrelevant titles (Fig. 1). Of the 25 papers eligible for full-text review, 17 were excluded, leaving eight papers representing eight studies for the review and six for the meta-analysis. 


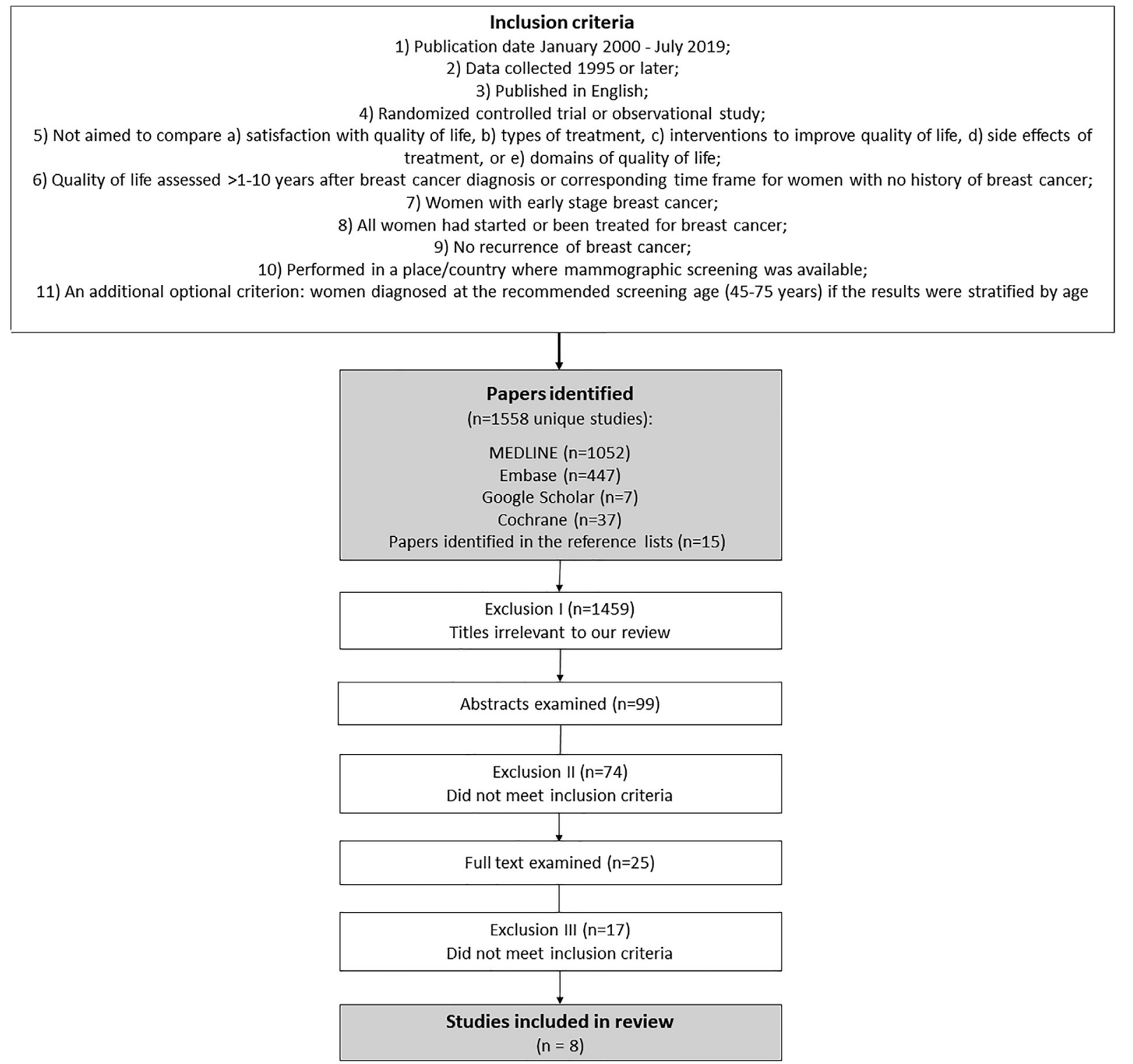

Fig. 1. Selection process with inclusion criteria

The reasons for the exclusion of the papers read in full are described in Appendix C, Table C1.

\section{Characteristics of the studies}

The eight studies included information about 6145 breast cancer survivors aged 21-80 years, diagnosed 1995-2012, and data collected during the period from 1996 to $2014^{21,35-41}$ (Tables 1 and 2 ). The screening coverage in the countries where the included studies were performed varied from $40.0 \%$ to $91.5 \% .{ }^{46,47}$ Two studies aimed to explore QoL among women with early-stage breast cancer, ${ }^{21,36}$ and the other included data from women with various stages of breast cancer at diagnosis. ${ }^{35,37-41}$ Five studies originated from Europe, ${ }^{35,36,38-40}$ one from Australia, ${ }^{37}$ and two from North America. ${ }^{21,41}$ Four studies used SF-36/RAND (Research and Development Corporation)-36, ${ }^{21,36,38,39}$ three EORTC studies used QLQ-C30, ${ }^{35,39,40}$ one study used FACT-G ${ }^{37}$ and one study used FACT-B. ${ }^{41}$ The studies reported $\mathrm{QoL}>1-10$ years after diagnosis or surgical treatment ${ }^{21,35-41}$ (Table 2 ). The reference groups included predominantly women aged $\geq 18$ years with no history of breast cancer. ${ }^{21,35-41}$ One study did not use a reference group but compared the results on QoL scores for early-stage breast cancer, locally advanced and metastatic breast cancer patients using FACT$\mathrm{B}^{41}$ (Table 3).

\section{QoL components}

In six studies, women followed $>1-10$ years postdiagnosis or since surgical treatment had lower mean scores for physical, cognitive, social and emotional functioning or well-being and higher mean scores for bodily pain compared with the reference groups ${ }^{21,35,36,38-40}$ (Table 2). However, in the study from Australia, women followed for 1.5 years postdiagnosis reported higher mean scores for social (23.4, 95\% CI: $22.6-24.2$ vs 19.8 , 95\% CI: 19.1-20.5) and functional well-being (22.5, 95\% CI: 21.7-23.2 vs 20.2 , 95\% CI: $19.5-20.9 ; P<0.05)$ compared with the reference group. ${ }^{37}$ In the study from the United States, women followed for $1.5-3$ years since surgical treatment 
Table 1

Characteristics of the studies included in the review.

\begin{tabular}{|c|c|c|c|c|c|c|c|c|c|}
\hline $\begin{array}{l}\text { First author, } \\
\text { publication year, ref } \\
\#\end{array}$ & Study aim & $\begin{array}{l}\text { Study country and } \\
\text { design }\end{array}$ & $\begin{array}{l}\text { Women } \\
\text { studied (age, n) }\end{array}$ & $\begin{array}{l}\text { Women } \\
\text { included in the } \\
\text { review (age, } \mathrm{n} \text { ) }\end{array}$ & Data source & $\begin{array}{l}\text { Data collection } \\
\text { method }\end{array}$ & $\begin{array}{l}\text { Diagnosis of breast } \\
\text { cancer (month, } \\
\text { year) }\end{array}$ & $\begin{array}{l}\text { Study period } \\
\text { (month, year) }\end{array}$ & Screening coverage (\%) \\
\hline Schou et al., $2005^{35}$ & $\begin{array}{l}\text { To compare HRQL } \\
\text { of women } \\
\text { diagnosed with } \\
\text { breast cancer with } \\
\text { the general female } \\
\text { population at } \\
\text { diagnosis and } 12 \\
\text { months since } \\
\text { surgical treatment } \\
\text { ( } \geq 14 \text { months } \\
\text { postdiagnosis) }\end{array}$ & $\begin{array}{l}\text { Norway; } \\
\text { longitudinal cohort } \\
\text { study }\end{array}$ & $\begin{array}{l}\text { Age } 21-78 \\
\text { years }(\mathrm{n}=161)\end{array}$ & $\begin{array}{l}\text { Age } 21-78 \\
\text { years }(\mathrm{n}=161)\end{array}$ & $\begin{array}{l}\text { Ullevål } \\
\text { University } \\
\text { Hospital }\end{array}$ & $\begin{array}{l}\text { Self-reported } \\
\text { questionnaire }\end{array}$ & $2002-2003$ & $2003-2004$ & $91.5^{47}$ \\
\hline $\begin{array}{l}\text { Van Gestel et al., } \\
2007^{36}\end{array}$ & $\begin{array}{l}\text { To compare the } \\
\text { HRQL, perceived } \\
\text { disease impact and } \\
\text { risk perception of } \\
\text { recurrence and } \\
\text { dying of breast } \\
\text { cancer in patients } \\
\text { with DCIS and EIBC } \\
2-3 \text { years } \\
\text { posttreatment }\end{array}$ & $\begin{array}{l}\text { The Netherlands; } \\
\text { cross-sectional } \\
\text { study }\end{array}$ & $\begin{array}{l}\text { Age } 30-80 \\
\text { years }(n=135)\end{array}$ & $\begin{array}{l}\text { Age } 50-69 \\
\text { years }(\mathrm{n}=75)\end{array}$ & $\begin{array}{l}\text { Eindhoven } \\
\text { Cancer Registry } \\
\text { of the } \\
\text { Comprehensive } \\
\text { Cancer Centre } \\
\text { South }\end{array}$ & $\begin{array}{l}\text { Self-reported } \\
\text { questionnaire }\end{array}$ & $\begin{array}{l}\text { January } 2002 \text { to } \\
\text { December } 2003\end{array}$ & May to June 2005 & $85.0^{61}$ \\
\hline $\begin{array}{l}\text { DiSipio et al., } \\
2008^{37}\end{array}$ & $\begin{array}{l}\text { To describe the } \\
\text { HRQL among breast } \\
\text { cancer survivors at } \\
6,12 \text { and } 18 \\
\text { months } \\
\text { postdiagnosis } \\
\text { compared with the } \\
\text { general female } \\
\text { population in } \\
\text { Queensland }\end{array}$ & $\begin{array}{l}\text { Australia; cohort } \\
\text { study }\end{array}$ & $\begin{array}{l}\text { Age } 20-74 \\
\text { years }(n=287)\end{array}$ & $\begin{array}{l}\text { Age } 50-74 \\
\text { years }(n=193)\end{array}$ & $\begin{array}{l}\text { Brisbane, } \\
\text { Queensland } \\
\text { and } \\
\text { Queensland } \\
\text { Cancer Registry }\end{array}$ & $\begin{array}{l}\text { Self-reported } \\
\text { questionnaire }\end{array}$ & $\begin{array}{l}\text { January to } \\
\text { December } 2002\end{array}$ & 2002-2004 & $58.0^{46}$ \\
\hline Klein et al., $2011^{39}$ & $\begin{array}{l}\text { To compare QoL of } \\
\text { breast cancer } \\
\text { survivors } 5 \text { and } 10 \\
\text { years since } \\
\text { diagnosis with QoL } \\
\text { of healthy controls }\end{array}$ & $\begin{array}{l}\text { France; cross- } \\
\text { sectional study }\end{array}$ & $\begin{array}{l}\text { Aged }<54 \text { and } \\
75+\text { years } \\
(\mathrm{n}=652)\end{array}$ & $\begin{array}{l}\text { Age }<54 \text { and } \\
75+\text { years } \\
\text { diagnosed } 10 \\
\text { years ago } \\
(\mathrm{n}=210)\end{array}$ & $\begin{array}{l}\text { Population- } \\
\text { based cancer } \\
\text { registries of } \\
\text { Bas-Rhin } \\
\text { (North-Eastern } \\
\text { France), } \\
\text { Calvados } \\
\text { (North- } \\
\text { Western } \\
\text { France), and } \\
\text { Doubs (Eastern } \\
\text { France) }\end{array}$ & $\begin{array}{l}\text { Self- reported } \\
\text { questionnaire }\end{array}$ & 1995 & 2005 & $40.0^{62}$ \\
\hline Jeffe et al., $2012^{21}$ & $\begin{array}{l}\text { To examine } \\
\text { changes in QoL in a } \\
\text { cohort of incident } \\
\text { early-stage breast } \\
\text { cancer and of } \\
\text { women with no } \\
\text { history of breast } \\
\text { cancer (controls) }\end{array}$ & $\begin{array}{l}\text { U.S.; longitudinal } \\
\text { case-control study }\end{array}$ & $\begin{array}{l}\text { Age } 40+\text { years } \\
(\mathrm{n}=549)\end{array}$ & $\begin{array}{l}\text { Age } 40+\text { years } \\
(\mathrm{n}=549)^{\mathrm{a}}\end{array}$ & $\begin{array}{l}\text { Siteman Cancer } \\
\text { Center at } \\
\text { Barnes-Jewish } \\
\text { Hospital, } \\
\text { Washington, } \\
\text { and St Louis } \\
\text { University } \\
\text { School of } \\
\text { Medicine }\end{array}$ & $\begin{array}{l}\text { Computer-assisted } \\
\text { telephone } \\
\text { interviews }\end{array}$ & $\begin{array}{l}\text { Information not } \\
\text { available }\end{array}$ & $\begin{array}{l}\text { October } 2003 \text { to } \\
\text { June } 2007\end{array}$ & $71.4^{63}$ \\
\hline $\begin{array}{l}\text { Browall et al., } \\
2013^{38}\end{array}$ & $\begin{array}{l}\text { To compare HRQL } \\
\text { in postmenopausal }\end{array}$ & & $\begin{array}{l}\text { Age } 55-80 \\
\text { years }(n=102)\end{array}$ & $\begin{array}{l}\text { Age } 55-80 \\
\text { years }(n=102)\end{array}$ & $\begin{array}{l}\text { Sahlgrenska } \\
\text { University }\end{array}$ & $\begin{array}{l}\text { Self- reported } \\
\text { questionnaire }\end{array}$ & 2003-2005 & $2003-2010$ & $70.0^{64 c}$ \\
\hline
\end{tabular}


women with breast Sweden;

cancer receiving

adjuvant treatment

longitudinal cohort

after surgery and

study

five years

posttreatmen

population

To explore in detail whether and to

what extent

restrictions in

breast cancer

survivors persist in

the long run and

whether changes or

aggravations in Qo

occur over time

$\begin{array}{ll}\text { Hamer et al., } 2017^{41} & \begin{array}{l}\text { To examine the } \\ \text { symptom burden }\end{array}\end{array}$

Canada; cross-

and QoL of different

sectional study

patient groups

across the breast

Germany;
Koch et al., $2013^{40}$

Hospital:

Department of

Breast Surgery,

Gothenburg:

Karolinska

University

Hospital:

Department
Oncology,

Stockholm

Skövde

Hospital, Dep of

Surgery,

Skövde

Age 18-80

years or olde
$(\mathrm{n}=387)$

Age 50-64

Population-

study $\quad(\mathrm{n}=387)$

based study in

Self-reported

Saarland

October 1996 to

February 1998

1996-2010

$\mathrm{n} / \mathrm{a}^{\mathrm{d}}$

HROL health related quality of life; QoL quality of life.

a The study was included in the review as the age when women typically start screening in the United States is 40 years, and the mean age for breast cancer survivors and the reference group was 58.9 (standard deviation, SD: 10.7) years and 57.2 (SD: 10.6) years, respectively, indicating that the majority of the included women were aged $>50$ years at enrolment.

Screening coverage is the percentage for screening attendance among eligible groups of women for the period when breast cancer was diagnosed.

Data available solely for Stockholm county.

Information is not available. 
Table 2

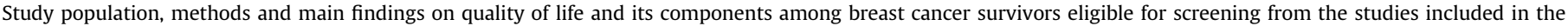
review.

\begin{tabular}{|c|c|c|c|c|c|c|}
\hline $\begin{array}{l}\text { First author, publication } \\
\text { year, ref \# }\end{array}$ & $\begin{array}{l}\text { Women included in the } \\
\text { review }(n, \%)^{b}\end{array}$ & $\begin{array}{l}\text { Breast cancer types/ } \\
\text { stages included in the } \\
\text { study }\end{array}$ & $\begin{array}{l}\text { Long-term } \\
\text { definition }\end{array}$ & Reference groups (n) & $\begin{array}{l}\text { Methods to evaluate } \\
\text { the QoL }\end{array}$ & $\begin{array}{l}\text { Main findings on QoL } \\
\text { and QoL components }\end{array}$ \\
\hline Schou et al., $2005^{35}$ & $\begin{array}{l}\text { Age } 21-78 \text { years } \\
(\mathrm{n}=161,100 \%)\end{array}$ & $\begin{array}{l}\text { Invasive BC stage I-II } \\
(\mathrm{n}=161)\end{array}$ & $\begin{array}{l}1 \text { year after } \\
\text { surgical } \\
\text { treatment }\end{array}$ & $\begin{array}{l}\text { Normal population } \\
\text { without diseases aged } \\
\text { 18-93 years (data from } \\
\text { 1998), using EORTC } \\
\text { QLQ-C30 }(\mathrm{n}=949)\end{array}$ & EORTC QLQ-C30 & $\begin{array}{l}\text { BCS had a lower mean } \\
\text { score for cognitive } \\
\left(82.0, \text { SD: } 18.3 \text { vs }^{\mathrm{a}} 86.6 \text {, }\right. \\
\left.\text { SD: } 19.2, P=0.008^{\mathrm{C}}\right) \\
\text { and social functioning } \\
(80.0, \mathrm{SD}: 23.4 \text { vs } 84.6 \text {, } \\
\left.\text { SD: } 22.4, P=0.009^{\mathrm{C}}\right) \\
\text { compared with the } \\
\text { reference group. }\end{array}$ \\
\hline Van Gestel et al., $2007^{36}$ & $\begin{array}{l}\text { Age } 50-69 \text { years } \\
(\mathrm{n}=75,64 \%)\end{array}$ & $\begin{array}{l}\text { DCIS }(\mathrm{n}=21) \text { and } \\
\text { invasive BC stage I, T1, } \\
\text { N0 and } \mathrm{MO}^{\mathrm{b}}(\mathrm{n}=54)\end{array}$ & $\begin{array}{l}1.5-3 \text { years } \\
\text { postdiagnosis }\end{array}$ & $\begin{array}{l}\text { Normal population } \\
\text { without diseases (data } \\
\text { from SF-36, collected } \\
\text { 1992-1996), from the } \\
\text { National study, } \\
\text { averages for SF-36 } \\
\text { domains, age and } \\
\text { gender adjusted }\end{array}$ & $\begin{array}{l}\text { RAND } \\
\text { SF-36 }\end{array}$ & $\begin{array}{l}\text { Women with DCIS had } \\
\text { a higher mean score for } \\
\text { bodily pain }(85.4 \text { vs } \\
\left.75.2, P=0.02^{\mathrm{f}}\right) \text { and } \\
\text { general mental health } \\
\left(77.8 \text { vs } 70.5, P=0.05^{\mathrm{f}}\right) \\
\text { compared with women } \\
\text { with early-stage BC, } \\
\text { and for bodily pain } \\
\left(85.4 \text { vs } 67.1, P<0.001^{\mathrm{f}}\right) \\
\text { and the physical } \\
\text { component scale }(49.6 \\
\left.\text { vs } 44.9, P<0.05^{\mathrm{f}}\right) \\
\text { compared with the } \\
\text { reference group. } \\
\text { Women with early- } \\
\text { stage BC had a higher } \\
\text { mean score for bodily } \\
\text { pain }(75.2 \text { vs } 67.1 \text {, } \\
\left.P<0.05^{\mathrm{f}}\right) \text { compared } \\
\text { with the reference } \\
\text { group. }\end{array}$ \\
\hline DiSipio et al., $2008^{37}$ & $\begin{array}{l}\text { Age } 50-74 \text { years } \\
(\mathrm{n}=193,74 \%)\end{array}$ & Unilateral invasive $\mathrm{BC}$ & $\begin{array}{l}1.5 \text { years ( } 18 \\
\text { months) } \\
\text { postdiagnosis }\end{array}$ & $\begin{array}{l}\text { Normal population } \\
\text { without diseases, aged } \\
\text { 30-74 years were } \\
\text { interviewed using QoL } \\
\text { data from } 2004 \\
(\mathrm{n}=675)\end{array}$ & FACT-G & $\begin{array}{l}\text { BCS had a higher mean } \\
\text { score for social }(23.4 \text {, } \\
95 \% \text { confidence } \\
\text { interval, CI: } 22.6-24.2 \\
\text { vs } 19.8,95 \% \text { CI: } 19.1 \\
-20.5)^{g} \text { and functional } \\
\text { well-being ( } 22.5,95 \% \\
\text { CI: } 21.7-23.2 \text { vs } 20.2 \text {, } \\
95 \% \text { CI: } 19.5-20.9)^{g} \\
\text { compared with the } \\
\text { reference group. } \\
\text { BCS had a clinically } \\
\text { better mean score for } \\
\text { QoL }(91.0,95 \% \text { CI: } 88.9 \\
-93.1 \text { vs } 86.0,95 \% \text { CI: } \\
84.5-87.5)^{g} \text { compared } \\
\text { with the reference } \\
\text { group. }\end{array}$ \\
\hline Klein et al., $2011^{39}$ & $\begin{array}{l}\text { Age }<54-75+\text { years } \\
\text { diagnosed } 10 \text { years ago } \\
(n=210,100 \%)\end{array}$ & $\begin{array}{l}\text { BC with no treatment } \\
\text { during the last } 5 \text { years }\end{array}$ & $\begin{array}{l}10 \text { years } \\
\text { postdiagnosis }\end{array}$ & $\begin{array}{l}\text { Normal population } \\
\text { matched by age and } \\
\text { place of residency to } \\
\text { patients using QoL data } \\
\text { from } 2005(\mathrm{n}=1188)\end{array}$ & EORTC QLQ-C30 & $\begin{array}{l}\text { BCS had a lower mean } \\
\text { score for physical ( } 81.6 \\
\text { vs } 84.6) \text {, role }(80.3 \text { vs } \\
84.5) \text { and social }(85.8 \text { vs } \\
88.6) \text { functioning } \\
\text { compared with the } \\
\text { reference group } \\
(P<0.0001 \text { for all })^{\mathrm{d}} \text {. }\end{array}$ \\
\hline Jeffe et al., $2012^{21}$ & $\begin{array}{l}\text { Age } \geq 40 \text { years }(n=549 \\
100 \%)^{f}\end{array}$ & $\begin{array}{l}\text { DCIS }(\mathrm{n}=148) \text { and a } \\
\text { first primary stage } 0 \text {-IIA } \\
\text { breast cancer without } \\
\text { neoadjuvant } \\
\text { chemotherapy } \\
(\mathrm{n}=365)\end{array}$ & $\begin{array}{l}2 \text { years } \\
\text { following } \\
\text { definitive } \\
\text { surgical } \\
\text { treatment }\end{array}$ & $\begin{array}{l}\text { Normal population } \\
\text { frequency-matched by } \\
\text { age }(40-49,50-69, \\
\geq 70 \text { years) to patients } \\
\text { were interviewed } 2 \\
\text { years and } 2 \text { weeks after } \\
\text { normal/benign } \\
\text { screening }(n=547)\end{array}$ & $\begin{array}{l}\text { RAND } \\
\text { 36-Item } \\
\text { Health Survey } 1.0\end{array}$ & $\begin{array}{l}\text { Women with early- } \\
\text { stage BC had a lower } \\
\text { mean score for physical } \\
\text { functioning ( } 76.3, \text { SD: } \\
25.3 \text { vs } 83.8, \text { SD: } 20.2 \text { ) } \\
\text { and role limitations due } \\
\text { to physical functioning } \\
\text { ( } 70.3, \text { SD: } 41.8 \text { vs } 78.2 \text {, } \\
\text { SD: } 36.0 \text { ), and a higher } \\
\text { mean score for } \\
\text { emotional well-being } \\
\text { (82.2, SD: } 16.8 \text { vs } 79.0 \text {, } \\
\text { SD: } 16.9) \text { compared }\end{array}$ \\
\hline
\end{tabular}


Table 2 (continued)

\begin{tabular}{lllll}
\hline $\begin{array}{l}\text { First author, publication } \\
\text { year, ref \# }\end{array}$ & $\begin{array}{l}\text { Women included in the } \\
\text { review }(\mathrm{n}, \%)^{\mathrm{b}}\end{array}$ & $\begin{array}{l}\text { Breast cancer types/ } \\
\text { stages included in the } \\
\text { study }\end{array}$ & $\begin{array}{l}\text { Long-term } \\
\text { definition }\end{array}$ & $\begin{array}{l}\text { Reference groups (n) } \\
\text { Methods } \\
\text { the QoL }\end{array}$ \\
\hline Browall et al., $2013^{38}$ & $\begin{array}{l}\text { Age } 55-80 \text { years } \\
(\mathrm{n}=102,100 \%)\end{array}$ & $\begin{array}{l}\text { Invasive breast cancer } \\
\text { stage I-III }(\mathrm{n}=102)\end{array}$ & $\begin{array}{l}5 \text { years postdiagnosis } \\
\text { matched by age (55-80 }\end{array}$ & $\begin{array}{l}\text { Normal population } \\
\text { years) to patients using } \\
\text { QoL data from } 2003 \text { to } \\
2010(n=426)\end{array}$ \\
\hline
\end{tabular}

Koch et al., 2013 $40 \quad$ Age $50-64$ years $(\mathrm{n}=76,42 \%)$

$\begin{array}{ll}\text { Hamer et al., } 2017^{41} & \text { Age } 51-70 \text { years } \\ (\mathrm{n}=857,58 \%)\end{array}$ $\begin{array}{ll}\text { Hamer et al., } 2017^{41} & \text { Age } 51-70 \text { years } \\ (\mathrm{n}=857,58 \%)\end{array}$
Stage at diagnosis local, 10 years regional and distant $\mathrm{BC}$ postdiagnosis
DCIS $(\mathrm{n}=83)$, invasive $1-10$ years

BC T1-T2 $(\mathrm{n}=464)$,

$\mathrm{T} 2 \mathrm{~N} 3$ or $\mathrm{T}^{\mathrm{e}}(\mathrm{n}=214)$

and metastatic BC

$(\mathrm{n}=98)$

\begin{abstract}
Normal population
aged 18 to $65+$ years

selected by random-

route-technique,

interviewed 1998,

using EORTC QLQ-C30

$(\mathrm{n}=968)$
\end{abstract}

Comparison between

the $\mathrm{BC}$ groups (stages)
FACT-B

EORTC QLQ-C30

(1) with the reference

$\operatorname{group}\left(P<0.05^{\mathrm{h}}\right.$ for all $)$.

BCS had a higher mean

score in physical

functioning (78.7

SD:20.5 vs 67.8 ,

SD:27.0), physical role

functioning (77.9, SD:

33.9 vs 61.2 , SD: 43.0 ),

bodily pain (77.3, SD:

23.8 vs 64.8, SD:29.5),

vitality (70.5, SD: 20.9

vs 62.8 , SD: 25.0 ), social

functioning (88.8,

SD:20.9 vs 82.7 ,

SD:24.8) and mental

health (82.7, SD:18.7 vs

76.6, SD: 22.5)

compared with the

reference group

$\left(P<0.05^{\mathrm{h}}\right.$ for all $)$

BCS had a lower mean

score for physical (84.5,

standard error, SE: 2.0

vs 89.4 , SE: 0.9$)$, role

(74.0, SE: 3.8 vs 87.9,

SE: 1.3), emotional

(60.2, SE: 3.3 vs 77.4 ,

SE: 1.3$)$, cognitive (72.8,

SE: 3.6 vs 91.1 , SE: 1.2$)$

and social functioning

(79.9, SE: 3.3 vs 91.1,

SE: 1.2) compared with

the reference group

$\left(P<0.05^{\mathrm{i}}\right.$ for all).

No significant

differences in the

overall mean QoL score

were found for women

with different breast

cancer stages. The

overall mean QoL score

reduced by stage for

those aged $51-70$ years

(120.0, SD: 18.6 for

DCIS, 117.4, SD: 20.3 for

early-stage invasive $\mathrm{BC}$

112.6, SD: 20.8 for

locally advanced and

101.4, SD: 23.7 for

metastatic $\mathrm{BC})$.

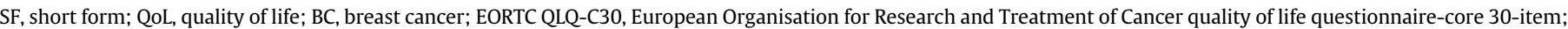

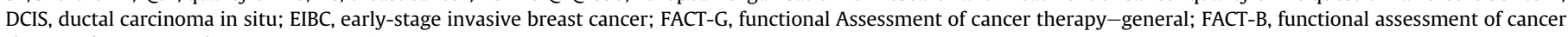
therapy-breast; BCS, breast cancer survivors.

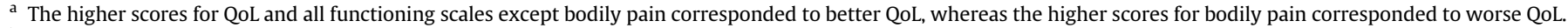

b Number and percentage of the entire sample of breast cancer survivors for each study.

c Based on $t$-tests.

d Adjusted for registry area, age, place of residence (urban/rural), marital status, education level, employment status, mean household monthly income, comorbidities and hospitalisation during the last 12 months (analysis of variance).

e Based on TNM Classification of Malignant Tumours (Union for International Cancer Control. J Brierley, M Gospodarowicz and C Wittekind. Wiley Blackwell, 2017).

${ }^{\mathrm{f}}$ Based on $t$-tests; standard deviation values were not available.

g $P$-values were not available; data were presented with $95 \%$ confidence intervals.

$\mathrm{h}$ Based on unadjusted analysis of variance.

${ }^{i}$ Based on age-adjusted analysis of variance.

reported better emotional well-being (82.2, SD: 16.8 vs 79.0, SD: $16.9 ; P<0.05)$ compared with the reference group. ${ }^{21}$ In the study from Sweden, women followed for 5 years postdiagnosis had higher mean scores for physical functioning
(78.7, SD: 20.5 vs 67.8 , SD: 27.0), social functioning (88.8, SD: 20.9 vs 82.7 , SD: 24.8$)$ and mental health (82.7, SD: 18.7 vs 76.6, SD: 22.5) compared with the reference group $(P<0.05$ for all). ${ }^{38}$ 


\begin{tabular}{|c|c|c|c|c|c|c|c|c|}
\hline $\begin{array}{l}\text { First author, } \\
\text { publication year, ref } \\
\#\end{array}$ & $\begin{array}{l}\text { Years since } \\
\text { diagnosis to } \\
\text { assessment }\end{array}$ & $\begin{array}{l}\text { Measurement } \\
\text { instrument }\end{array}$ & $\begin{array}{l}\text { Age of breast cancer } \\
\text { survivors }\end{array}$ & $\begin{array}{l}\text { Mean quality of life } \\
\text { of breast cancer } \\
\text { survivors }\end{array}$ & $\begin{array}{l}\text { Age of reference } \\
\text { group }\end{array}$ & $\begin{array}{l}\text { Type of the } \\
\text { reference group }\end{array}$ & $\begin{array}{l}\text { Mean quality of life } \\
\text { of reference group }\end{array}$ & $\begin{array}{l}P \text {-value for } \\
\text { comparison } \\
\text { between breast } \\
\text { cancer survivors } \\
\text { and reference } \\
\text { group }\end{array}$ \\
\hline Schou et al., $2005^{35}$ & $>1$ year $^{\mathrm{a}}$ & $\begin{array}{l}\text { EORTC QLQ-C30 (0 } \\
-100)\end{array}$ & $21-78$ years & $\begin{array}{l}75.7^{\mathrm{b}} \text { (standard } \\
\text { deviation, SD: } 21.4) \\
(\mathrm{n}=161)\end{array}$ & $18-93$ years & Healthy women & $\begin{array}{l}72.0 \text { (SD: 24.5) } \\
(\mathrm{n}=949)\end{array}$ & $0.28^{\mathrm{m}}$ \\
\hline $\begin{array}{l}\text { Van Gestel et al., } \\
2007^{36}\end{array}$ & $1.5-3$ years & $\begin{array}{l}\text { RAND SF-36 (0 } \\
-100)\end{array}$ & $50-69$ years & $\begin{array}{l}63.0^{c} \\
(\mathrm{n}=75)\end{array}$ & $50-69$ years & Healthy women & $\begin{array}{l}63.0 \\
\text { (not available) }\end{array}$ & - \\
\hline $\begin{array}{l}\text { DiSipio et al., } \\
2008^{37}\end{array}$ & 1.5 years & FACT-G $(0-108)$ & $50-74$ years & $\begin{array}{l}91.0(95 \% \\
\text { confidence interval, } \\
\text { CI: 88.9-93.1 })^{\mathrm{h}} \\
(\mathrm{n}=193)\end{array}$ & $30-74$ years & Healthy women & $\begin{array}{l}86.0(95 \% \text { CI: } 84.5 \\
-88.4)^{\mathrm{h}} \\
(\mathrm{n}=675)\end{array}$ & $\begin{array}{l}\text { Significant clinical } \\
\text { difference }^{j}\end{array}$ \\
\hline Klein et al., $2011^{39}$ & 10 years & $\begin{array}{l}\text { EORTC QLQ-C30 (0 } \\
-100)\end{array}$ & $<54-75+$ years & $\begin{array}{l}66.3^{g} \\
(\mathrm{n}=210)\end{array}$ & $<54-75+$ years & Healthy women & $\begin{array}{l}69.2 \\
(\mathrm{n}=1188)\end{array}$ & $0.0035^{k}$ \\
\hline Jeffe et al., $2012^{21}$ & 2 years $^{\mathrm{i}}$ & $\begin{array}{l}\text { RAND 36-Item } \\
\text { Health Survey } 1.0 \\
(0-100)\end{array}$ & $\geq 40$ years & $\begin{array}{l}68.0^{\mathrm{C}}(\mathrm{SD}: 22.6) \\
(\mathrm{n}=549)\end{array}$ & $\geq 40$ years & Healthy women & $\begin{array}{l}73.4 \text { (SD: 21.1) } \\
(\mathrm{n}=547)\end{array}$ & $0.0017^{k}$ \\
\hline $\begin{array}{l}\text { Browall et al., } \\
2013^{38}\end{array}$ & 5 years & SF-36 (0-100) & $55-80$ years & $\begin{array}{l}70.5^{\mathrm{f}}(\mathrm{SD}: 20.9) \\
(\mathrm{n}=102)\end{array}$ & $55-80$ years & Healthy women & $\begin{array}{l}62.7(\text { SD: } 25.0) \\
(\mathrm{n}=426-475)\end{array}$ & $<0.001^{\mathrm{k}}$ \\
\hline Koch et al., $2013^{40}$ & 10 years & $\begin{array}{l}\text { EORTC QLQ-C30 (0 } \\
-100)\end{array}$ & $50-64$ years & $\begin{array}{l}68.0^{\mathrm{e}} \text { (standard } \\
\text { error, SE: } 2.6) \\
(\mathrm{n}=76)\end{array}$ & 50-64 years & Healthy women & $\begin{array}{l}68.1 \text { (SE: 2.1) } \\
(\mathrm{n}=968)\end{array}$ & $0.86^{1}$ \\
\hline Hamer et al., $2017^{41}$ & $1-10$ years & FACT-B $(0-123)$ & $51-70$ years & $\begin{array}{l}110.5^{\mathrm{d}}(\text { SD: } 21.6) \\
(\mathrm{n}=857)\end{array}$ & - & - & - & - \\
\hline
\end{tabular}

a Time since surgical treatment was at least 12 months.

b Women with early stage (I-II) breast cancer.

d Women with early-stage invasive breast cancer.

e Women with all types of breast cancer including ductal carcinoma in situ.

e Women with all types of breast cancer
f Women with stage I-III breast cancer.

${ }_{\mathrm{g}}$ Adjusted for registry area, age, place of residence (urban/rural), marital status, education level, employment status, mean household monthly income, comorbidities and hospitalisation during the last 12 months (analysis of variance).

${ }^{\mathrm{h}}$ Mean health-related quality of life score for women with invasive breast cancer.

i Time since surgical treatment.

${ }^{\mathrm{j}}$ Based on $95 \%$ confidence intervals.

$k$ Based on unadjusted analysis of variance.

${ }^{1}$ Based on age-adjusted analysis of variance.

$m$ Based on linear regression analysis. 


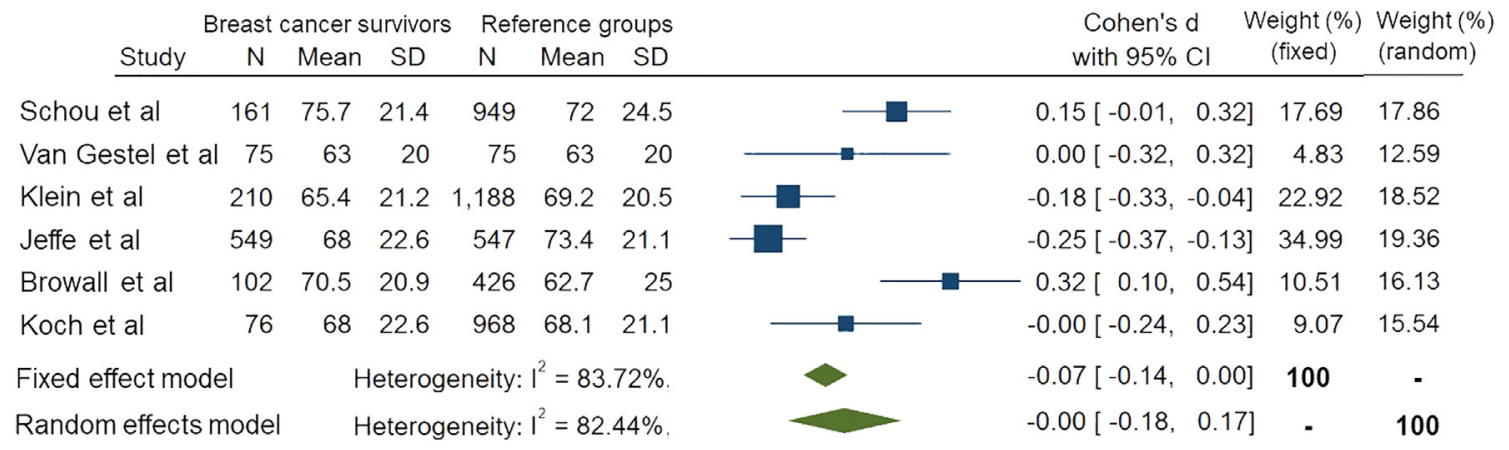

\begin{tabular}{lll}
\hline-5 & 0 & .5
\end{tabular}

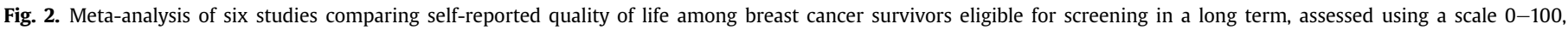
compared with the reference groups of women. N, number; SD, standard deviation.

\section{Quality of life}

A mean score for QoL among breast cancer survivors followed for $>1-10$ years postdiagnosis or since surgical treatment varied from 63.0 (on a scale of $0-100$ ) to 110.5 (on a scale of $0-123)^{37,38}$ (Table 3). Seven studies compared QoL among breast cancer survivors and the reference groups. ${ }^{21,35-40}$ In three studies, the mean score for QoL did not differ between breast cancer survivors followed for $>1-10$ years postdiagnosis and the reference groups. ${ }^{35,36,40}$ In two studies, breast cancer survivors reported a higher mean score for QoL compared with the reference groups (91.0, 95\% CI: $88.9-93.1$ vs $86.0,95 \%$ CI $84.5-87.5$ on a scale of 0-108; and 70.5, SD: 20.9 vs 62.7, SD: 25.0 on a scale of $0-100$, $P<0.05$, respectively). ${ }^{37,38}$ In two studies, breast cancer survivors reported a lower mean score for QoL compared with the reference groups (68.0, SD: 22.6 vs 73.4, SD: 21.1 on a scale of $0-100 ; P<0.05$; and 66.3 vs 69.2 on a scale of $0-100, P<0.05$, respectively). ${ }^{21,40}$ In the study from Canada, the mean score for QoL for women aged 50-71 years with all types of invasive breast cancer was 110.5, SD: 21.6 , on a scale of $0-123 .{ }^{41}$

Pooled effect measured from six studies presented by a Cohen's d was -0.07 (95\% CI -0.14 to 0.00$)$ with $\mathrm{I}^{2}=82.4 \%$, and $-0.00(95 \%$ CI -0.18 to 0.17 ) with $\mathrm{I}^{2}=83.7 \%$ for the fixed effect and random effects models, respectively (Fig. 2). The funnel plot did not show any small-study effect, as no differences between the comparison groups were found in the small studies (Appendix D, Fig. D1).

\section{Discussion}

Our review identified a mean score for long-term QoL among breast cancer survivors eligible for mammographic screening and followed for $>1-10$ years since diagnosis to vary from 63.0 (on a scale of $0-100$ ) to 110.5 (on a scale of $0-123$ ). ${ }^{37,38}$ The studies showed better, $^{37,38}$ similar, $^{35,36,40}$ or poorer ${ }^{21,39}$ QoL among breast cancer survivors compared with women with no history of breast cancer (the reference group). The effect size model based on six studies using a scale from 0 to 100 to measure the mean QoL did not show any statistically significant differences between breast cancer survivors and women with no history of breast cancer, eligible for mammographic screening.

The better results for breast cancer survivors could be explained by the study settings, implying that most of the women had early-stage breast cancer diagnosed in screening programmes. ${ }^{37,38}$ Furthermore, women attending screening might be healthier and have a higher breast awareness than non-attendees. ${ }^{48-51}$ On the other side, women usually consider screening as a check and might thus not be prepared for a diagnosis of breast cancer in contrast to women seeking mammography due to symptoms. However, the better results might also have been associated with a relatively short follow-up (1.5-5 years since diagnosis). ${ }^{37,38}$ Furthermore, no matching by age with the reference group and the possibility of various chronic diseases in the reference group might have resulted in higher scores for QoL and the functioning components for the breast cancer survivors compared with the reference group in one of the studies. ${ }^{37,52}$

Similar results for breast cancer survivors and women with no history of breast cancer were found in three studies. ${ }^{35,36,40}$ In one of these studies, the length of the follow-up might have been too short to show any differences ( $\geq 14$ months since diagnosis). ${ }^{35}$ In the other study, the data from the reference group were obtained for a long time before the study start, which might have limited health perceptions, as different health awareness, treatments and methods of care were present in that period compared with the study period. ${ }^{36}$ Furthermore, a small number of women $(n=75)$ in each group and the length of follow-up of 1.5-3 years might have contributed to the lack of differences. In the other study, the differences in the disease-specific symptom burden implied a less favourable pattern for breast cancer survivors compared with the reference group. ${ }^{40}$ However, a small number of women $(n=76)$ with breast cancer vs a large number of women in the reference group $(\mathrm{n}=968)$, and using a 10-year follow-up with a study period 1996-1998, when the treatment recommendations differed from those used in 2000s, might have led to the lack of differences in QoL scores between the groups. ${ }^{40,41}$ The similar results on QoL, but clinically relevant deterioration in symptoms and several QoL components, could be explained by the response shift or the adaptive mechanisms influencing the overall QoL perception, but not functioning or symptom burden. ${ }^{40,53}$

The poorer QoL among breast cancer survivors compared with that of women with no history of breast cancer was expected. ${ }^{21,39}$ However, such results were shown in the study from the United States, including women aged $\geq 40$ years, where younger women were known to have more advanced breast cancer compared with older women. ${ }^{21}$ The main reasons for this are more aggressive treatment associated with high proliferative aggressive tumours and a stronger impact of treatment on the everyday life of women aged $<50$ years. ${ }^{54,55}$ On the other side, screening of women in their $40 \mathrm{~s}$ is more common in the United States compared with Europe and might mirror the insurance coverage. ${ }^{56}$ The poorer results of the QoL of breast cancer survivors eligible for screening compared with the reference group in a large population-based study with a 
10-year follow-up might be considered the most relevant result of this review, ${ }^{39}$ assuming that the majority of women in the study population were in the age group 54-75 years. However, the study started in 1995 and was associated with more aggressive treatment for women with early-stage breast cancer and might have impacted the lower scores of QoL among breast cancer survivors compared with women with no history of breast cancer. ${ }^{26,30}$

Various study settings and periods, numbers of women and lengths of follow-up might have contributed to the results of the meta-analysis, showing no difference between breast cancer survivors and women with no history of breast cancer, eligible for screening. ${ }^{21,35,36,38-40}$

\section{Limitations of the studies included in the review}

The quality of the reporting in the included studies was rather low with regard to the CONSORT-PRO criteria, as the main limitations included non-reporting the baseline outcomes and underreporting the characteristics of comparison groups ${ }^{21,36-41}$ (Appendix B). Bias due to confounding was observed in three studies and was associated with different types of treatment and therefore QoL perceptions among women with early-stage breast cancer, and not adjusting for possible comorbidities not pertaining to breast cancer. ${ }^{36,38,41}$ Selection bias was found in all included studies and was associated with small sample sizes and differences in the age ranges between the reference groups and breast cancer survivors, different social and race status of participants and non-participants, and including solely women who participated in all follow-ups. ${ }^{21,35-41}$ Bias due to missing data was presented in four studies and indicated low response and lack of information about loss due to follow-up, underreporting of the poorest cases, and association of the data collection methods with the respondents who could be reached by telephone and whose participation might be associated with insurance coverage..$^{21,35-41}$ Bias in measurement outcome was observed in all included studies and was associated with the lack of baseline information, using only one time point to measure the outcome, and limitations of the selfreported questionnaire and computer-assisted telephone interviews. $^{21,35-38,40,41}$ Bias in the selection of the reported results was found in three studies and included the older data collection period for the reference (1992-1996) vs the study sample (2002-2003), use of clinical but not statistical significance and comparing the findings for women diagnosed at different points of time between 1996 and 2010. ${ }^{36,37,40}$ According to the assessment of the risk of bias, the studies by Schou et al. and by Klein et al. could be considered the most reliable, as these did not show any serious risk of bias. ${ }^{35,39}$

\section{Limitations of the review and meta-analysis}

Women eligible for screening were aged $21-80$ years in our review and solely four studies included women of typical screening age in Europe, at diagnosis. ${ }^{36,37,40,41}$ The overall age range of the review might have been associated with lower scores for QoL and functioning scales due to the inclusion of women aged $<45$ years and $>75$ years, ${ }^{41,54,57}$ who might have reported poorer QoL compared with women of screening age. ${ }^{58-60}$ However, in all the included studies, the majority of the women were of the typical screening age (45-75 years) at diagnosis, except for the study from Norway and the United States, where it was not possible to differentiate women by age groups. The inclusion of studies performed between 1995 and 2018 might have resulted in the poorer scores for breast cancer survivors in the studies, started in 1995-1996 compared with those started in the 2000s, due to improved breast cancer treatment, including reconstructive and breast conserving surgery and neoadjuvant chemotherapy. ${ }^{26}$ Furthermore, the pure impact of participation in mammographic screening was not investigated in this review. However, based on the screening coverage in the included studies, the majority of the women might have been diagnosed due to screening. ${ }^{46,47,61-65}$ Future research is needed to compare QoL between women with screen-detected breast cancer and women with no history of breast cancer in the areas, where mammographic screening is available.

We have not included a study using FACT-G (0-108) questionnaire in the meta-analysis, which might have contributed to the less favourable results for breast cancer survivors' QoL. SF-36/RAND and EORTC-QLQ-C30 were included in the meta-analysis on the equal basis because of the same measurement scale, $0-100$, despite their content differed. ${ }^{66}$ This could have resulted in overestimation as well as underestimation of the outcome. Furthermore, the differences in study design, length of follow-up, number of women included and periods when treatment was performed might have influenced the overall effect. Meta-regression was not performed because fewer than 10 studies were included. ${ }^{67}$

In conclusion, this review did not identify differences in QoL between women diagnosed with breast cancer and followed for >1-10 years compared with women with no history of breast cancer among those eligible for mammographic screening.

\section{Author statements}

\section{Ethical approval}

None declared.

\section{Funding}

The study was supported by a grant (N 2019/FO244363) from Stiftelsen Dam to Nataliia Moshina via the Norwegian Women's Public Health Association. The funding source was not involved in the study.

\section{Competing interests}

All the authors declare no conflict of interest.

\section{Authors' contributions}

Each author can take responsibility for the content of the article. The literature search was performed by all the authors of the article. All the authors have made substantial contribution to the conception and design of the study, acquisition of data, analyses and interpretation of findings. N.M. worked with drafting the article, and all the authors contributed to revising it critically for important intellectual content. The final approval of the version to be submitted was given by all authors.

\section{Appendix A. Supplementary data}

Supplementary data to this article can be found online at https://doi.org/10.1016/j.puhe.2021.08.008.

\section{References}

1. Ferlay J. Global cancer observatory: cancer today. Lyon, France: International Agency for Research on Cancer; 2018.

2. IARC handbook of cancer prevention volume 7. Breast cancer screening. IARC Press; 2002.

3. Broeders M, Paci E. The balance sheet of benefits and harms of breast cancer population-based screening in Europe: outcome research, practice and future challenges. Women's Health (London, England) 2015;11(6):883-90.

4. Paci E, Broeders M, Hofvind S, Duffy SW. The benefits and harms of breast cancer screening. Lancet (London, England) 2013;381(9869):800-1. 
5. Brodersen J, Siersma VD. Long-term psychosocial consequences of falsepositive screening mammography. Ann Fam Med 2013;11(2):106-15.

6. Heleno B, Siersma VD, Brodersen J. Diagnostic invasiveness and psychosocial consequences of false-positive mammography. Ann Fam Med 2015;13(3): 242-9.

7. Brett J, Bankhead C, Henderson B, Watson E, Austoker J. The psychological impact of mammographic screening. A systematic review. Psycho Oncol 2005; 14(11):917-38.

8. Lampic C, Thurfjell E, Bergh J, Sjoden PO. Short- and long-term anxiety and depression in women recalled after breast cancer screening. Eur J Cancer (Oxford, England : 1990) 2001;37(4):463-9.

9. Ganz PA. Impact of tamoxifen adjuvant therapy on symptoms, functioning, and quality of life. J Natl Cancer Inst Monogr 2001;(30):130-4.

10. Ganz PA, Desmond KA, Leedham B, Rowland JH, Meyerowitz BE, Belin TR. Quality of life in long-term, disease-free survivors of breast cancer: a follow-up study. J Natl Cancer Inst 2002;94(1):39-49.

11. Ganz PA, Kwan L, Stanton AL, Krupnick JL, Rowland JH, Meyerovitz BE, et al. Quality of life at the end of primary treatment of breast cancer: first results from the moving beyond cancer randomized trial. J Natl Cancer Inst 2004;96(5): 376-87.

12. Ganz PA, Rowland JH, Desmond K, Meyerowitz BE, Wyatt GE. Life after breast cancer: understanding women's health-related quality of life and sexual functioning. J Clin Oncol 1998;16(2):501-14. official journal of the American Society of Clinical Oncology.

13. Ganz PA, Rowland JH, Meyerowitz BE, Desmond KA. Impact of different adjuvant therapy strategies on quality of life in breast cancer survivors. Recent Results Cancer Res 1998;152:396-411.

14. Falk R, Hofvind S, Skaane P, Haldorsen T. Overdiagnosis among women attending a population-based mammography screening program. Int J Cancer 2013;133(3):705-12.

15. Puliti D, Duffy SW, Miccinesi G, de Koning H, Lynge E, Zappa M, et al. Overdiagnosis in mammographic screening for breast cancer in Europe: a literature review. J Med Screen 2012;19(Suppl 1):42-56.

16. Chu WO, Dialla PO, Roignot P, Bone-Lepinoy MC, Poillot ML, Coutant C, et al. Determinants of quality of life among long-term breast cancer survivors. Qual Life Res 2016;25(8):1981-90. an international journal of quality of life aspects of treatment, care and rehabilitation.

17. Tomich PL, Helgeson VS. Five years later: a cross-sectional comparison of breast cancer survivors with healthy women. Psycho Oncol 2002;11(2):154-69.

18. Groenvold M. Health-related quality of life in early breast cancer. Dan Med Bull 2010;57(9):B4184.

19. Goyal NG, Levine BJ, Van Zee KJ, Naftalis E, Avis NE. Trajectories of quality of life following breast cancer diagnosis. Breast Canc Res Treat 2018;169(1):163-73.

20. Lidgren M, Wilking N, Jonsson B, Rehnberg C. Health related quality of life in different states of breast cancer. Qual Life Res: 2007;16(6):1073-81. an international journal of quality of life aspects of treatment, care and rehabilitation.

21. Jeffe DB, Pérez M, Liu Y, Collins KK, Aft RL, Schootman M. Quality of life over time in women diagnosed with ductal carcinoma in situ, early-stage invasive breast cancer, and age-matched controls. Breast Canc Res Treat 2012;134(1):379-91.

22. Euhus DM, Addae JK, Snyder CF, Canner JK. Change in health-related quality of life in older women after diagnosis of a small breast cancer. Cancer 2019;125(11):1807-14.

23. de Koning HJ, van Ineveld BM, van Oortmarssen GJ, de Haes JC, Collette HJ, Hendriks JH, et al. Breast cancer screening and cost-effectiveness; policy alternatives, quality of life considerations and the possible impact of uncertain factors. Int J Cancer 1991;49(4):531-7.

24. Zahl PH, Kalager M, Suhrke P, Nord E. Quality-of-life effects of screening mammography in Norway. Int J Cancer 2020;146(8):2104-12.

25. Moher D, Shamseer L, Clarke M, Ghersi D, Liberati A, Petticrew M, et al. Preferred reporting items for systematic review and meta-analysis protocols (PRISMA-P) 2015 statement. Syst Rev 2015;4:1.

26. Heilat GB, Brennan ME, French J. Update on the management of early-stage breast cancer. Aust J Gen Pract 2019;48(9):604-8.

27. Kaarlola A, Tallgren M, Pettilä V. Long-term survival, quality of life, and qualityadjusted life-years among critically ill elderly patients. Crit Care Med 2006;34(8):2120-6

28. Pierre CS, Dassonville O, Chamorey E, Poissonnet G, Ettaiche M, Santini J, et al. Long-term quality of life and its predictive factors after oncologic surgery and microvascular reconstruction in patients with oral or oropharyngeal cancer. Eur Arch Oto-Rhino-Laryngol 2014;271(4):801-7.

29. World Health Organisation. International classification of functioning, disability and health (ICF). 2001. https://www.who.int/standards/classifications/ international-classification-of-functioning-disability-and-health\#: :text=ICF\% 20is\%20the\%20WHO\%20framework,and\%20measure\%20health\%20and\% 20disability. [Accessed 25 December 2020].

30. Jensen M-B, Ejlertsen B, Mouridsen HT, Christiansen P. Improvements in breast cancer survival between 1995 and 2012 in Denmark: the importance of earlier diagnosis and adjuvant treatment. Acta Oncol 2016;55(sup2):24-35.

31. Biesheuvel C, Weigel S, Heindel W. Mammography screening: evidence, history and current practice in Germany and other European countries. Breast Care 2011;6(2):104-9.

32. Schunemann HJ, Lerda D, Quinn C, Follmann M, Alonso-Coello P, Rossi PG, et al. Breast cancer screening and diagnosis: a synopsis of the European breast guidelines. Ann Intern Med 2019;172(1):46-56.
33. Methley AM, Campbell S, Chew-Graham C, McNally R, Cheraghi-Sohi S. PICO, PICOS and SPIDER: a comparison study of specificity and sensitivity in three search tools for qualitative systematic reviews. BMC Health Serv Res 2014;14:579.

34. Recommendations on breast cancer screening 24/11/2016. 2016. http://ecibc.jrc. ec.europa.eu/recommendations/details/4. [Accessed 27 December 2016].

35. Schou I, Ekeberg Ø, Sandvik L, Hjermstad MJ, Ruland CM. Multiple predictors of health-related quality of life in early stage breast cancer. Data from a year follow-up study compared with the general population. Qual Life Res 2005;14(8):1813-23. an international journal of quality of life aspects of treatment, care and rehabilitation.

36. van Gestel YR, Voogd AC, Vingerhoets AJ, Mols F, Nieuwenhuijzen GAP, Repelaer van Driel OJ, et al. A comparison of quality of life, disease impact and risk perception in women with invasive breast cancer and ductal carcinoma in situ. Eur J Cancer (Oxford, England : 1990) 2007;43(3):549-56.

37. DiSipio T, Hayes S, Newman B, Janda M. Health-related quality of life 18 months after breast cancer: comparison with the general population of Queensland, Australia. Support Care Cancer 2008;16(10):1141-50. official journal of the Multinational Association of Supportive Care in Cancer.

38. Browall M, Ostlund U, Henoch I, Wengstrom Y. The course of Health Related Quality of Life in postmenopausal women with breast cancer from breast surgery and up to five years post-treatment. Breast (Edinburgh, Scotland) 2013;22(5):952-7.

39. Klein D, Mercier M, Abeilard E, Puyraveau M, Danzon A, Dalstein V, et al. Longterm quality of life after breast cancer: a French registry-based controlled study. Breast Canc Res Treat 2011;129(1):125-34.

40. Koch L, Jansen L, Herrmann A, Stegmaier C, Holleczek B, Singer S, et al. Quality of life in long-term breast cancer survivors - a 10-year longitudinal populationbased study. Acta Oncol (Stockholm, Sweden) 2013;52(6):1119-28.

41. Hamer J, McDonald R, Zhang L, Verma S, Leahey A, Ecclestone C, et al. Quality of life (QOL) and symptom burden (SB) in patients with breast cancer. Support Care Cancer 2017;25(2):409-19.

42. Cohen J. Statistical power analysis for the behavioral sciences. Hillsdale, NJ: Lawrence Earlbaum Associates; 1988.

43. Higgins JP, Thompson SG, Deeks JJ, Altman DG. Measuring inconsistency in meta-analyses. Br Med J 2003;327(7414):557-60.

44. Higgins JPTAD, Sterne JAC. Chapter 8: assessing risk of bias in included studies. In: Higgins JPT, Churchill R, Chandler J, Cumpston MS, editors. Cochrane handbook for systematic reviews of interventions version 5.2.0. Cochrane; 2017.

45. Calvert M, Blazeby J, Altman DG, Revicki DA, Moher D, Brundage MD. Reporting of patient-reported outcomes in randomized trials: the CONSORT PRO extension. Jama 2013;309(8):814-22.

46. Youlden DRCS, Baade PD. Current status of female breast cancer in Queensland, 1982 to 2006 Brisbane. Queensland: Viertel Centre for Research in Cancer Control Cancer Council Queensland; 2009.

47. Lynge E, Braaten $\mathrm{T}$, Njor SH, Olsen AH, Kumle M, Waaseth $\mathrm{M}$, et al Mammography activity in Norway 1983 to 2008. Acta Oncol (Stockholm, Sweden) $2011 ; 50(7): 1062-7$.

48. Biesheuvel C, Czene K, Orgeas CC, Hall P. The role of mammography screening attendance and detection mode in predicting breast cancer survival-is there added prognostic value? Cancer Epidemiol 2011;35(6):545-50.

49. Manjer AR, Emilsson UM, Zackrisson S. Non-attendance in mammography screening and women's social network: a cohort study on the influence of family composition, social support, attitudes and cancer in close relations. World J Surg Oncol 2015;13:211.

50. Lagerlund M, Widmark C, Lambe M, Tishelman C. Rationales for attending or not attending mammography screening-a focus group study among women in Sweden. Eur J Cancer Prev 2001;10(5):429-42. the official journal of the European Cancer Prevention Organisation (ECP).

51. Lagerlund M, Sontrop JM, Zackrisson S. Psychosocial factors and attendance at a population-based mammography screening program in a cohort of Swedish women. BMC women's health 2014;14(1):33.

52. DiSipio T, Rogers C, Newman B, Whiteman D, Eakin E, Fritschi L, et al. The Queensland Cancer Risk Study: behavioural risk factor results. Aust N Z J Public Health 2006;30(4):375-82.

53. Sprangers MA, Schwartz CE. Integrating response shift into health-related quality of life research: a theoretical model. Soc Sci Med 1999;48(11):1507-15.

54. Lee HB, Han W. Unique features of young age breast cancer and its management. J Breast Cancer 2014;17(4):301-7.

55. Brandt J, Garne JP, Tengrup I, Manjer J. Age at diagnosis in relation to survival following breast cancer: a cohort study. World J Surg Oncol 2015;13(1): 33.

56. Nelson RA, Bostanci Z, Jones V, Mortimer J, Polverini A, Taylor L, et al. Insurance status predicts survival in women with breast cancer: results of breast and cervical cancer treatment program in California. Ann Surg Oncol 2020. https:// doi.org/10.1245/s10434-019-08116-x.

57. Cohen L, Hamer J, Helwig C, Fergus K, Kiss A, Mandel R, et al. Formal evaluation of PYNK: breast cancer program for young women-the patient perspective. Curr Oncol 2016;23(2):e102-8.

58. Derks MG, de Glas NA, Bastiaannet E, de Craen AJM, Portielje JEA, van de Velde $\mathrm{CJH}$, et al. Physical functioning in older patients with breast cancer: a prospective cohort study in the TEAM trial. Oncologist 2016;21(8):946-53.

59. Leach CR, Bellizzi KM, Hurria A, Reeve BB. Is it my cancer or am i just getting older?: impact of cancer on age-related health conditions of older cancer survivors. Cancer 2016;122(12):1946-53. 
60. Bellury L, Pett MA, Ellington L, Beck SL, Clark JC, Stein KD. The effect of aging and cancer on the symptom experience and physical function of elderly breast cancer survivors. Cancer 2012;118(24):6171-8.

61. Louwman WJ, van de Poll-Franse LV, Fracheboud J, Roukema JA, Coebergh JW. Impact of a programme of mass mammography screening for breast cancer on socio-economic variation in survival: a population-based study. Breast Canc Res Treat 2007; 105(3):369-75.

62. Gianino MM, Lenzi J, Bonaudo M, Fantini MP, Siliquini R, Ricciardi W, et al. Organized screening programmes for breast and cervical cancer in 17 EU countries: trajectories of attendance rates. BMC Public Health 2018;18(1):1236.

63. Lian M, Jeffe DB, Schootman M. Racial and geographic differences in mammography screening in St. Louis City: a multilevel study. J Urban Health 2008;85(5):677-92.
64. Lind H, Svane G, Kemetli L, Törnberg S. Breast cancer screening program in Stockholm county, Sweden - aspects of organization and quality assurance. Breast Care (Basel, Switzerland) 2010;5(5):353-7.

65. Cancer Quality Council of Ontario. Breast cancer screening participation. 2019.

66. Apolone G, Filiberti A, Cifani S, Ruggiata R, Mosconi P. Evaluation of the EORTC QLQ-C30 questionnaire: a comparison with SF-36 Health Survey in a cohort of Italian long-survival cancer patients. Ann Oncol 1998;9(5):549-57. official journal of the European Society for Medical Oncology/ESMO.

67. Borenstein MHL, Higgins JPT, Rothstein $\mathrm{H}$. Introduction to meta-analysis. 3rd ed. ed. United Kingdom: John Wiley \& Sons; 2011. 